\title{
INFLUÊNCIA DO ESPAÇAMENTO NAS CARACTERÍSTICAS ENERGÉTICAS DE ESPÉCIES ARBÓREAS EM PLANTIOS DE CURTA ROTAÇÃO ${ }^{1}$
}

Elder Eloy², Braulio Otomar Caron ${ }^{3}$, Dimas Agostinho da Silva², Denise Schmidt ${ }^{3}$, Rômulo Trevisan ${ }^{4}$, Alexandre Behling ${ }^{5}$ e Elvis Felipe Elli ${ }^{3}$

\begin{abstract}
RESUMO - Este trabalho teve como objetivo determinar o poder calorífico superior (PCS) das árvores e a massa específica básica ponderada $\left(\rho_{\mathrm{b} \text { pond }}\right)$ da madeira de Acacia mearnsii De Wild, Eucalyptus grandis W. Hill ex Maiden, Mimosa scabrella Benth e Ateleia glazioviana Baill distribuídas em diferentes espaçamentos em plantio: 2,0 x 1,0 m; 2,0 x 1,5 m; 3,0 x 1,0 m; e 3,0 x 1,5 m, nas idades de 1 e 3 anos. O estudo foi conduzido em um experimento localizado no Município de Frederico Westphalen, RS, em delineamento experimental de blocos completos casualizados com três repetições, no esquema de parcelas subdivididas. A determinação do PCS das árvores foi realizada a partir da ponderação dos valores obtidos em cada compartimento (madeira, casca, galho e folha), por meio de bomba calorimétrica. A determinação da $\rho_{\mathrm{b} \text { pond }}$ foi realizada a partir da ponderação dos valores verificados dos discos coletados em diferentes posições ao longo do tronco, usando-se o método da balança hidrostática e massa seca. O PCS de todas as espécies no primeiro ano após o plantio foi superior ao do terceiro ano, destacando-se a Acacia mearnsii com as maiores médias. A $\rho_{\mathrm{b} \text { pond }}$ não apresentou tendência sistemática de aumento ou redução ao longo do tempo e as espécies Acacia mearnsii e Ateleia glazioviana, os maiores valores. Os diferentes espaçamentos de plantio não induziram a variação do PCS e da $\rho_{\mathrm{b} \text { pond }}$ nas espécies estudadas, não sendo verificada tendência sistemática positiva ou negativa em relação ao espaço vital proporcionado pelo espaçamento.
\end{abstract}

Palavras-chave: Propriedade da madeira; Energia da biomassa; Espaçamento de plantio.

\section{INFLUENCE OF SPACING ON ENERGY CHARACTERISTICS OF TREE SPECIES ON SHORT ROTATION PLANTING}

\begin{abstract}
This study aimed to determine the higher heating value (PCS) of trees and basic density weighted $\left(\rho_{\text {b pond }}\right)$ of wood of Acacia mearnsii De Wild, Eucalyptus grandis W. Hill ex Maiden, Mimosa scabrella Benth and Ateleia glazioviana Baill distributed in different spacings in planting: $2.0 \times 1.0 \mathrm{~m}, 2.0 \times 1.5 \mathrm{~m}, 3.0$ $\times 1.0 \mathrm{~m}$ and $3.0 \times 1.5 \mathrm{~m}$, at ages of 1 and 3 years. The study was conducted in an experiment in the city of Frederico Westphalen, State of RS, in experimental design of randomized complete block design with three replications in a split plots. The determination of the PCS of the trees was made from the weighting of the values obtained in each compartment (wood, bark, twig and leaf) using calorimeter bomb. The determination of $\rho_{\text {bpond }}$ was performed from the weighting of the values recorded on discs collected at different positions along the stem, using the method of hydrostatic balance and dry mass. The PCS of all species in the first year after planting was higher than the third year, highlighting the Acacia mearnsii with the highest averages.
\end{abstract}

\footnotetext{
${ }^{1}$ Recebido em 29.09.2012 aceito para publicação em 30.04.2014.

${ }^{2}$ Departamento de Engenharia e Tecnologia Florestal, Universidade Federal do Paraná, Paraná, Brasil. E-mail: <eloyelder@yahoo.com.br> e $<$ dimas.agostinho.silva@gmail.com>.

${ }^{3}$ Departamento de Agronomia, Universidade Federal de Santa Maria, Frederico Westphalen, RS, Brasil. E-mail: <otomarcaron@yahoo.com.br>, <schmidtbr2000@yahoo.com.br>e <elvisfelipeelli@yahoo.com>.

${ }^{4}$ Departamento de Engenharia Florestal, Universidade Federal de Santa Maria, Frederico Westphalen, RS, Brasil. E-mail: <romulo_trevisan@yahoo.com.br>.

${ }^{5}$ Departartamento de Manejo Florestal, Universidade Federal do Paraná, Paraná, Brasil. E-mail: <alexandre.behling@yahoo.com.br>.
} 
The $\rho_{\text {bpond }}$ had no systematic trend of increase or decrease over time, and the species Acacia mearnsii and Ateleia glazioviana showed the highest values. The different planting spacings did not induce the change in PCS and $\rho_{\text {bond }}$ in the species studied and it was not verified a positive or negative systematic trend in relation to the living space provided by the spacing.

Keywords: Weibull, Eucalyptus, Artificial Intelligence.

\section{INTRODUÇÃO}

No cenário energético nacional, a lenha ocupa posição importante, tratando-se de um recurso natural renovável que pode ter sua produção sustentável e não apresentar o caráter poluidor de outras fontes fósseis. Inegavelmente, a madeira apresenta papel fundamental em termos de estratégias ligadas à produção e ao uso de energia, sendo evidente a retomada do seu consumo para tal finalidade, atividade que vem crescendo continuamente ao longo da última década no Brasil.

A valorização da biomassa como insumo energético moderno surgiu na década de 1970, com as crises do petróleo em 1973 e 1979 (MÜLLER, 2005). No Brasil, em 1940, aproximadamente $80 \%$ da energia consumida era proveniente da madeira. Em 1969, esse percentual caiu para 33,7\% e, em 2011, para 9,7\% (BRASIL, 2012). Apesar desse decréscimo significativo da participação da madeira como fonte energética, em termos quantitativos o consumo não teve alteração significativa, evidenciando que há um mercado cativo para a utilização de biomassa florestal como fonte de energia.

Em se tratando de materiais combustíveis, como é o caso da madeira como recurso dendroenergético, esse deve basear-se, entre outros, no conhecimento do seu potencial para produção de biomassa e do seu poder calorífico superior (PCS), sendo essa uma das características mais importantes. O poder calorífico da madeira consiste na quantidade de energia na forma de calor liberada pela combustão completa de uma unidade de massa do material combustível (ÇENGEL; BOLES, 2006).

Há duas maneiras de se expressar o poder calorífico de um combustível, sendo o PCS e o poder calorífico inferior (PCI). O PCS é aquele obtido na bomba calorimétrica a partir do combustível seco, em que a combustão se efetua em volume constante e no qual a água formada é condensada e o calor resultante, recuperado. Já o PCI é aquele calculado a partir do PCS, sendo a energia efetivamente disponível por unidade de massa de combustível após deduzir as perdas com

Revista Árvore, Viçosa-MG, v.38, n.3, p.551-559, 2014 a evaporação da água, ou seja, considera o teor de umidade a que se encontra o material combustível. Em termos de pesquisa e comparação de combustíveis, o PCS é mais usual (CINTRA, 2009).

Na literatura, encontram-se trabalhos que envolvem a obtenção do PCS de diferentes espécies florestais, tanto nativas quanto exóticas. Quirino et al. (2005), ao revisarem a literatura sobre diferentes espécies florestais tropicais, encontraram valor médio de PCS igual a $4.732 \mathrm{kcal} \mathrm{kg}^{-1}$, variando do limite inferior de $3.350 \mathrm{kcal} \mathrm{kg}^{-1}$ ao limite superior de $5.260 \mathrm{kcal} \mathrm{kg}^{-1}$. Esses mesmos autores relataram valores de PCS para a espécie Mimosa scabrella de 4.589 a $4.890 \mathrm{kcal} \mathrm{kg}^{-1}$ e para Eucalyptus grandis de 4.501 a $4.701 \mathrm{kcal} \mathrm{kg}^{-1}$, estando dentro da faixa encontrada por Vale et al. (2000), que verificaram para essa espécie valores médios de PCS de $4.641 \mathrm{kcal} \mathrm{kg}^{-1}$ aos 7 anos de idade. Já Baggio (2002), estudando a espécie Ateleia glazioviana, observou um PCS de $4.450 \mathrm{kcal} \mathrm{kg}^{-1}$. Silva et al. (2012), trabalhando com Acacia mearnsii, Eucalyptus grandis, Mimosa scabrella e Ateleia glazioviana em povoamentos com 1 ano de idade, encontraram um PCS médio de $4.482 \mathrm{kcal}$ $\mathrm{kg}^{-1}, 4.346 \mathrm{kcal} \mathrm{kg}^{-1}, 4.511 \mathrm{kcal} \mathrm{kg}^{-1}$ e $4.492 \mathrm{kcal} \mathrm{kg}^{-1}$, respectivamente.

A massa específica básica $\left(\rho_{b}\right)$ representa a concentração de massa por volume na madeira e influencia a velocidade da queima durante a produção direta de energia, assim como a densidade do carvão vegetal oriundo dessas madeiras, por isso é interessante que as madeiras apresentem $\rho_{\mathrm{b}}$ altas. Alguns autores assumem que essa faixa seja acima de $0,65 \mathrm{~g} \mathrm{~cm}^{-3}$ (VALE, 2002). Da mesma forma, Lopes (2003) assumiu uma $\rho_{\mathrm{b}}$ para Eucalyptus grandis de $0,460 \mathrm{~g} \mathrm{~cm}^{-3}$, e Carvalho (1998) encontrou valores que variaram de 0,560 e 0,850 $\mathrm{g} \mathrm{cm}^{-3}$ em Acacia mearnsii, enquanto Jankowsky et al. (1990) relataram valores entre 0,510 e 0,610 $\mathrm{g} \mathrm{cm}^{-3}$ em Mimosa scabrella. Já Mattos (2000) observou uma $\rho_{\mathrm{b}}$ de $0,530 \mathrm{~g} \mathrm{~cm}^{-3}$ para Ateleia glazioviana. Dessa forma, estudo da $\rho_{\mathrm{b}}$ da madeira em diferentes espécies florestais torna-se de fundamental importância, por ser o resultado de uma complexa combinação dos seus 
constituintes internos. Esse parâmetro tecnológico, além de ser muito utilizado, apresenta alta eficiência para expressar a qualidade da madeira, a fim de prognosticar sua variação em diferentes usos, nos diversos segmentos da atividade industrial, devido à íntima relação com várias outras propriedades e, também, por ser de fácil determinação (WASHUSEN et al., 2005).

As causas para variação na $\rho_{\mathrm{b}}$ podem ser atribuídas às diferentes condições ambientais e genéticas de cada árvore ou, ainda, em função de diferentes idades, condições ambientais e fatores genéticos. Essas influências podem comprometer o uso de espécies florestais, pois ocasionam modificações em seu desempenho durante o uso energético, assim como na qualidade de seus produtos, o que não é interessante quando se almejam altos desempenhos e produtos com qualidades padronizadas (TRUGILHO et al., 1996).

Quando se trata da comparação de características anatômicas da madeira, autores como Cunha et al. (1989) afirmaram que não há correlação significativa entre o PCS e a $\rho_{\mathrm{b}}$ da madeira. No entanto, madeiras que apresentam maior $\rho_{b}$ implicam maior concentração de massa por volume, o que é interessante durante a aplicação energética desse material.

Tais divergências encontradas na literatura relacionadas com as características energéticas, muitas vezes, confundem a escolha de espécies florestais direcionadas à produção de energia. Assim, neste trabalho, procurou-se avaliar espécies que são difundidas no Sul do país, sendo o Eucalyptus grandis e Acacia mearnsii espécies exóticas muito cultivadas, assim como a Mimosa scabrella e Ateleia glazioviana, que são nativas características da Região Sul.

Dentro desse contexto, este trabalho teve como intuito a determinação do poder calorífico superior das árvores e da massa específica básica ponderada da madeira das espécies florestais Acacia mearnsii De Wild, Eucalyptus grandis W. Hill ex Maiden, Mimosa scabrella Benth e Ateleia glazioviana Baill, distribuídas em diferentes espaçamentos e idades de plantio.

\section{MATERIAL E MÉTODOS}

\subsection{Caracterização da área em estudo}

O trabalho foi realizado no experimento que está localizado em área pertencente ao Laboratório de Agroclimatologia (LAGRO), vinculado à Universidade
Federal de Santa Maria (UFSM), Campus do Centro de Educação Superior Norte do Rio Grande do Sul (CESNORS), sob coordenadas geográficas de $27^{\circ} 22^{\prime \prime S}$ e $53^{\circ} 25^{\prime \prime} \mathrm{W}$, a $480 \mathrm{~m}$ de altitude, no Município de Frederico Westphalen, RS.

Segundo a classificação climática de Köppen, o clima da região é subtropical úmido com verão quente (Cfa). A área experimental está distante de Iraí a aproximadamente $30 \mathrm{~km}$, sendo o município tomado como referência para os dados de classificação climática. Conforme proposta de Maluf (2000), Iraí apresenta clima de tipo subtemperado subúmido, sendo a temperatura média anual de $18,8^{\circ} \mathrm{C}$ e, a temperatura média do mês mais frio, de $13,3^{\circ} \mathrm{C}$.

O experimento foi instalado utilizando o delineamento de blocos completos casualizados. Os blocos foram caracterizados por um fatorial $4 \times 4$, ou seja, quatro espécies florestais (Acacia mearnsii De Wild, Eucalyptus grandis W. Hill ex Maiden, Mimosa scabrella Benth e Ateleia glazioviana Baill) e quatro espaçamentos (2,0 x 1,0 m, 2,0 x 1,5 m, 3,0 x 1,0 m e 3,0 x 1,5 m) em três repetições, no esquema de parcelas subdivididas, em que a parcela foi representada pelo espaçamento mais espécie e a subparcela pela idade de aferição dos dados. O bloco contempla 16 unidades experimentais, que apresentam 45 plantas cada, distribuídas em cinco linhas, estando as unidades experimentais divididas em quatro subparcelas, compostas por três plantas cada.

O solo predominante na área é do tipo Latossolo Vermelho distrófico típico e, no seu preparo para o plantio das mudas, foram realizadas as operações de aração e gradagem, sendo o plantio feito de forma manual, em setembro de 2008 .

Com a finalidade de atender aos objetivos do projeto, no $1^{\circ}, 3^{\circ}, 5^{\circ}$ e $7^{\circ}$ anos foram realizadas avaliações destrutivas de cada unidade experimental. Dessa forma, neste trabalho foram utilizados os dados das avaliações destrutivas coletados no $1^{\circ}$ ano (2009) e no $3^{\circ}$ ano (2011) de experimento.

\subsection{Determinação da massa específica básica ponderada $\left(\rho_{\text {b pond }}\right)$}

Para o estudo da $\rho_{b}$, foram retirados discos com aproximadamente $2 \mathrm{~cm}$ de espessura, nas seguintes posições no tronco: $0 \%$ (base), 1,30 m (diâmetro à altura do peito - DAP) e $25 \%$, 50\% e $75 \%$ da altura total da

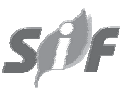

Revista Árvore, Viçosa-MG, v.38, n.3, p.551-559, 2014 
árvore. Os discos foram numerados de acordo com sua posição relativa no tronco e localização experimental. Em seguida, foram acondicionados em sacos plásticos e transportados para o Laboratório de Agroclimatologia UFSM/CESNORS, onde foram marcadas e seccionadas duas cunhas simetricamente opostas.

Os procedimentos realizados nas avaliações da massa específica básica madeira $\left(\rho_{\mathrm{b}}\right)$, em $\mathrm{g} \mathrm{cm}^{-3}$, seguiram os procedimentos da norma COPANT 30:1:004 (COPANT, 1971). Nesses procedimentos, determinaram-se o volume verde (Vu), em cm³ , e a massa seca (Mo), em g, utilizando a seguinte expressão:

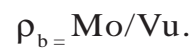

A $\rho_{b}$ da madeira em cada posição relativa do tronco foi determinada pela média dos valores das duas cunhas. Em seguida, calculou-se a massa específica básica ponderada ( $\rho_{\mathrm{b} \text { pond }}$ ) em função do volume de cada árvore, em $\mathrm{g} \mathrm{cm}^{-3}$. Esse cálculo se baseia na ponderação dos valores de $\rho_{\mathrm{b}}$ obtida em cada posição relativa ( $\left.\rho_{\mathrm{b} \text { ”i” }}\right)$, em $\mathrm{g} \mathrm{cm}^{-3}$; os volumes sem casca correspondentes entre duas posições sucessivas $\left(\mathrm{v}_{1}, \mathrm{v}_{2}, \mathrm{v}_{\mathrm{i}}\right)$, em $\mathrm{m}^{3}$; com o volume total sem casca da árvore $\left(\mathrm{v}_{\mathrm{s}}\right)$, em $\mathrm{m}^{3}$, definido pelo método de Smalian (FINGER, 2002). Assim, a $\rho_{\mathrm{b} \text { pond }}$ foi definida pela seguinte expressão:

$$
\rho_{\mathrm{b} \text { pond }}=\left(\left(\left(\left(\rho_{\mathrm{b} 0 \%}+\rho_{\mathrm{bDAP}}\right) / 2\right) \mathrm{v}_{1}\right)+\left(\left(\left(\rho_{\mathrm{bDAP}}+\rho_{\mathrm{b} 25 \%}\right) /\right.\right.\right.
$$
2) $\left.\left.v_{1}\right)+\left(\left(\left(\tilde{n}_{b i}+\tilde{n}_{b i+1}\right) / 2\right) v_{i}\right)\right) / v_{s}$.

\subsection{Determinação do poder calorífico superior (PCS)}

Para determinação do PCS das árvores, foram retirados discos com aproximadamente $2 \mathrm{~cm}$ de espessura, nas seguintes posições no tronco: $0 \%$ (base), 1,30 m (diâmetro à altura do peito - DAP) e 25\%, 50\%, $75 \%$ e $100 \%$ da altura total, num total de seis discos por árvore, sendo posteriormente separada a casca da madeira de cada disco.

As amostras de galhos e folhas foram coletadas de forma estratificada na planta, ou seja, nos estratos inferior, médio e superior da copa das árvores, com a finalidade de obtenção de um material mais homogêneo que representasse toda a expansão da copa. Essas foram identificadas e levadas para secagem em estufa de circulação e renovação do ar para obtenção da matéria seca. As amostras de madeira, casca, folha e galho foram secas a $103^{\circ} \mathrm{C}$ até peso constante. Posteriormente, foram moídas em moinho de facas, com peneira de 40 mesh, visando à obtenção de um material mais fino e uniforme, sendo esse procedimento realizado em duplicata por amostra.

A determinação do PCS dos diferentes compartimentos (madeira, casca, galho e folha) das árvores foi realizada no Laboratório de Energia de Biomassa Florestal do Departamento de Engenharia e Tecnologia Florestal da Universidade Federal do Paraná (UFPR), utilizando bomba calorimétrica adiabática modelo IKA-WERNE; C5000, de acordo com a norma ABNT/ NBR 8633/84.

Em seguida, calculou-se o poder calorífico superior ponderado ( $\mathrm{PC}_{\text {pond }}$ ) em função da quantidade de biomassa de cada compartimento da árvore. Esse cálculo está baseado na ponderação dos valores de poder calorífico superior obtidos em cada compartimento ( $\mathrm{PC}_{, \mathrm{j}}$, ), em kcal.kg-1; os pesos correspondentes a cada compartimento ( $\mathrm{P}_{\text {Madeira }}, \mathrm{P}_{\text {Casca }}, \mathrm{P}_{\text {Galho }}$ e $\left.\mathrm{P}_{\text {Folha }}\right)$, em kg; com o peso total da árvore $\left(\mathrm{P}_{\text {Total }}\right)$, em $\mathrm{kg}$. Assim, o $\mathrm{PC}_{\text {pond }}$ foi definido pela seguinte expressão:

$$
\begin{aligned}
& \mathrm{PC}_{\text {pond }}=\left(\left(\mathrm{PC}_{\text {Madeira }} * \mathrm{P}_{\text {Madeirar }}\right)+\left(\mathrm{PC}_{\text {Casca }} * \mathrm{P}_{\text {Casca }}\right)+\right. \\
& \left.\left(\mathrm{PC}_{\text {Galho }} * \mathrm{P}_{\text {Galho }}\right)+\left(\mathrm{PC}_{\text {Folha }} * \mathrm{P}_{\text {Folha }}\right)\right) / \mathrm{P}_{\text {Total }} \cdot
\end{aligned}
$$

\subsection{Análise dos dados}

Os dados foram submetidos à análise estatística através do Software Statistical Analysis System (SAS, 2003), em que se determinaram a análise de variância e o teste de Tukey a 5\% de probabilidade de erro.

\section{RESULTADOS}

A análise de variância revelou diferença no PCS e na $\rho_{\mathrm{b}}$ somente para a interação dos dois anos avaliados com as quatro espécies arbóreas estudadas, não sendo observada essa característica nos quatro espaçamentos diante dos dois períodos nos quatro espaçamentos em relação às quatro espécies arbóreas estudadas (Tabela 1). Dessa forma, pode-se observar que as diferentes densidades de plantio (2,0 x 1,0 m; 2,0 x 1,5 m; 3,0 x 1,0 m; e 3,0 x 1,5 m) não influenciaram significativamente as quatro espécies arbóreas (Acacia mearnsii, Eucalyptus grandis, Mimosa scabrella e Ateleia glazioviana), tanto no primeiro ano (2009) quanto no terceiro ano (2011) após o plantio.

Quando analisado o PCS das árvores tanto no primeiro ano de avaliação quanto no terceiro, observou-se que a Acacia mearnsii apresentou os maiores valores 
Tabela 1 - Análise de variância do poder calorífico superior (PCS) e da massa específica básica $\left(\rho_{\mathrm{b}}\right)$ da madeira das espécies arbóreas distribuídas nos diferentes espaçamentos, em diferentes anos após o plantio, no Município de Frederico Westphalen, RS.

Table 1 - Analysis of variance for the higher heating value (PCS) and basic density $\left(\rho_{b}\right)$ of wood in the tree species distributed in different spacings, in different years after planting, in the city of Frederico Westphalen, RS.

\begin{tabular}{|c|c|c|c|c|}
\hline \multicolumn{5}{|c|}{ Efeito principal } \\
\hline \multirow{2}{*}{\multicolumn{2}{|c|}{ Fator de estudo }} & \multirow[t]{2}{*}{ GL } & \multicolumn{2}{|c|}{ Quadrado médio } \\
\hline & & & PCS & $\rho_{\mathrm{b}}$ \\
\hline Espécie & & 3 & $223042 *$ & $0,0598^{*}$ \\
\hline Espaçam & & 3 & $7908^{\mathrm{ns}}$ & $0,0018^{\text {ns }}$ \\
\hline Espécie & & 9 & 4672 ns & 0,0009 ns \\
\hline \multicolumn{5}{|c|}{ * Significativo à probabilidade de erro tipo A } \\
\hline Ano & & 1 & 1205568* & $0,0211^{*}$ \\
\hline Ano $x$ es & & 3 & $51823 *$ & $0,0182 *$ \\
\hline Ano $x$ es & & 3 & 7272 ns & $0,0011^{\mathrm{ns}}$ \\
\hline Ano $x$ es & & 9 & 4989 ns & $0,0007^{\mathrm{ns}}$ \\
\hline \multicolumn{5}{|c|}{ * Significativo à probabilidade de erro tipo B } \\
\hline Coeficie & (\%) & & $86 \%$ & $61 \%$ \\
\hline Coeficie & & & 1,67 & 7,03 \\
\hline \multicolumn{5}{|c|}{ Efeito simples } \\
\hline \multicolumn{5}{|c|}{ Ano $x$ espécie } \\
\hline \multirow[t]{2}{*}{ Ano } & 1 & 3 & $146548 *$ & $0,0063^{*}$ \\
\hline & 3 & 3 & $128318 *$ & $0,0701 *$ \\
\hline \multirow[t]{4}{*}{ Espécie } & A. mearnsii & 1 & $375500 *$ & $0,0083^{*}$ \\
\hline & M. scabrella & 1 & $416172 *$ & 0,0002 ns \\
\hline & E. grandis & 1 & $523272 *$ & $0,0045^{*}$ \\
\hline & A. glazioviana & 1 & $46095 *$ & $0,0599 *$ \\
\hline
\end{tabular}

Em que * = significativo a 5\% de probabilidade de erro, conforme a distribuição de Fisher; ${ }^{\text {ns }}=$ não significativo a $5 \%$ de probabilidade de erro, conforme a distribuição de Fisher.

em todos os espaçamentos de plantio, em comparação com as outras espécies. Em contrapartida, a espécie Ateleia glazioviana apresentou as menores médias de PCS no primeiro ano após o plantio. Já no Eucalyptus grandis foi observada essa característica no terceiro ano (Tabela 2).

Pode-se observar, de maneira geral, que os PCS de todas as espécies distribuídas nos diferentes espaçamentos no primeiro ano foram superiores aos do terceiro ano. O motivo dessa discrepância pode ter sido influenciado pelo fato de que cada ano que passa a planta cresce e adquire mais lignina, o que favorece o aumento do PCS. Essa propriedade, observada na Tabela 3, apresentou valores para a espécie Eucalyptus grandis que variaram de 4.241 a $4.653 \mathrm{kcal} \mathrm{kg}^{-1}$, para Mimosa scabrella de 4.349 a $4.679 \mathrm{kcal} \mathrm{kg}^{-1}$, para Acacia mearnsii de 4.442 a $4.828 \mathrm{kcal} \mathrm{kg}^{-1}$ e para Ateleia glazioviana de 4.440 a $4.586 \mathrm{kcal} \mathrm{kg}^{-1}$.

Quando analisada a $\rho_{\mathrm{b} \text { pond }}$ das árvores, tanto no primeiro ano quanto no terceiro após o plantio, observou-se que não ocorreu variação sistemática de aumento ou redução ao longo do tempo. A Ateleia glazioviana apresentou os maiores valores, em comparação com as outras espécies. Em contrapartida, o Eucalyptus grandis apresentou as menores médias de $\rho_{\mathrm{b} \text { pond }}$ no primeiro e no terceiro ano após o plantio (Tabela 3).

As $\rho_{\mathrm{b} \text { pond }}$ observadas apresentaram valores da espécie Eucalyptus grandis que variaram de 0,365 a 0,391 $\mathrm{g} \mathrm{cm}^{-3}$ para Mimosa scabrella, de 0,388 a 0,411 $\mathrm{g} \mathrm{cm}^{-3}$ para Acacia mearnsii de 0,402 a $0,447 \mathrm{~g} \mathrm{~cm}^{-3}$ e para Ateleia glazioviana de 0,399 a 0,489 $\mathrm{g} \mathrm{cm}^{-3}$ (Tabela 3).

\section{DISCUSSÃO}

Ao avaliarem os valores de PCS em diferentes níveis de adubação para Eucalyptus grandis e Acacia mangium, Vale et al. (2000) verificaram que, para o eucalipto e a acácia-negra, os valores de PCS foram, em média, de $4.641 \mathrm{kcal} \mathrm{kg}^{-1}$ e $4.619 \mathrm{kcal} \mathrm{kg}^{-1}$, respectivamente, aos 7 anos de idade. Esses valores 
Tabela 2 - Poder calorífico superior (PCS), em kcal kg-1 das espécies arbóreas distribuídas nos diferentes espaçamentos, em diferentes anos após o plantio, no Município de Frederico Westphalen, RS.

Table 2 - Higher heating value (PCS), in $\mathrm{kcal} \mathrm{kg}^{-1}$, of the tree species distributed in different spacings, in different years after planting, in the city of Frederico Westphalen-RS.

\begin{tabular}{|c|c|c|c|c|}
\hline \multirow[t]{2}{*}{ Espécie } & \multicolumn{4}{|c|}{ Espaçamento (m) } \\
\hline & $2,0 \times 1,0$ & $2,0 \times 1,5$ & $3,0 \times 1,0$ & $3,0 \times 1,5$ \\
\hline \multicolumn{5}{|c|}{ Ano 1} \\
\hline A. mearnsii & 4726 a & 4788 a & 4792 а & 4828 a \\
\hline M. scabrella & $4647 \mathrm{ab}$ & $4658 \mathrm{~b}$ & $4631 \mathrm{~b}$ & $4679 \mathrm{~b}$ \\
\hline E. grandis & 4531 с & 4600 bc & 4539 bc & $4653 \mathrm{~b}$ \\
\hline A. glazioviana & 4586 bc & $4533 \mathrm{c}$ & 4476 c & 4520 c \\
\hline CV (\%) & 1,81 & 2,33 & 2,98 & 2,70 \\
\hline \multicolumn{5}{|c|}{ Ano 3} \\
\hline A. mearnsii & 4558 а & 4442 a & 4560 a & 4577 а \\
\hline M. scabrella & 4372 b & $4349 \mathrm{ab}$ & $4406 \mathrm{~b}$ & $4435 \mathrm{~b}$ \\
\hline E. grandis & $4338 \mathrm{~b}$ & $4289 \mathrm{~b}$ & $4241 \mathrm{c}$ & 4273 c \\
\hline A. glazioviana & $4448 \mathrm{ab}$ & 4441 a & $4440 \mathrm{ab}$ & $4436 \mathrm{~b}$ \\
\hline CV (\%) & 2,20 & 1,71 & 2,98 & 2,80 \\
\hline
\end{tabular}

Médias seguidas por letras iguais, na coluna, não diferem entre si, a 5\% de probabilidade de erro, conforme a distribuição de Tukey.

Tabela 3 -Massa específica básica ponderada $\left(\rho_{\mathrm{b} \text { pond }}\right)$ da madeira, em $\mathrm{g} \mathrm{cm}^{-3}$, das espécies arbóreas distribuídas nos diferentes espaçamentos, em diferentes anos após o plantio, no Município de Frederico Westphalen, RS.

Table 3 - Basic Density weighted ( $\rho_{\text {bpond }}$ ) of wood, in $\mathrm{g} \mathrm{cm}^{-3}$, of the tree species distributed in different spacings, in different years after planting, in the city of Frederico Westphalen, RS.

\begin{tabular}{lcccc}
\hline Espécie & \multicolumn{5}{c}{ Espaçamento (m) } \\
\cline { 2 - 5 } & $2,0 \times 1,0$ & $2,0 \times 1,5$ & $3,0 \times 1,0$ & $3,0 \times 1,5$ \\
\hline A. mearnsii & $0,415 \mathrm{a}$ & $0,402 \mathrm{a}$ & $0,406 \mathrm{~b}$ & $0,405 \mathrm{a}$ \\
M. scabrella & $0,388 \mathrm{~b}$ & $0,409 \mathrm{ab}$ & $0,405 \mathrm{~b}$ & $0,406 \mathrm{a}$ \\
E. grandis & $0,382 \mathrm{~b}$ & $0,387 \mathrm{~b}$ & $0,390 \mathrm{~b}$ & $0,391 \mathrm{a}$ \\
A. glazioviana & $0,417 \mathrm{a}$ & $0,423 \mathrm{a}$ & $0,440 \mathrm{a}$ & $0,399 \mathrm{a}$ \\
CV (\%) & 4,52 & 3,70 & 5,15 & 1,72 \\
\hline \multicolumn{7}{c}{ mearnsii } & & & $0,414 \mathrm{a}$ & $0,436 \mathrm{ab}$ \\
M. scabrella & $0,447 \mathrm{ab}$ & $0,407 \mathrm{~b}$ & $0,411 \mathrm{~b}$ \\
E. grandis & $0,405 \mathrm{~b}$ & $0,414 \mathrm{~b}$ & $0,365 \mathrm{c}$ & $0,384 \mathrm{~b}$ \\
A. glazioviana & $0,367 \mathrm{c}$ & $0,396 \mathrm{~b}$ & $0,489 \mathrm{a}$ & $0,486 \mathrm{a}$ \\
CV (\%) & $0,472 \mathrm{a}$ & $0,371 \mathrm{c}$ & 12,32 & 10,11 \\
\hline
\end{tabular}

Médias seguidas por letras iguais, na coluna, não diferem entre si, a 5\% de probabilidade de erro, conforme a distribuição de Tukey.

estão entre uma faixa de valores semelhantes aos encontrados por Howard (1973), que observou um PCS variando na faixa de 4.600 a $4.800 \mathrm{kcal} \mathrm{kg}^{-1}$.

A partir da análise, no PCS das espécies em estudo, verificou-se que estão de acordo com os relatados da literatura. Jara (1989) relatou valores de $4.550 \mathrm{kcal} \mathrm{kg}^{-1}$ para Acacia decurrens (acácia-negra) aos 5 anos de idade e $4.790 \mathrm{kcal} \mathrm{kg}^{-1}$ para o Eucalyptus grandis aos 10 anos de idade. Já Pereira et al. (2000), estudando várias espécies de eucalipto, observaram valores que variaram de $5.080 \mathrm{kcal} \mathrm{kg}^{-1}$ para Eucalyptus camaldulensis com 6 anos de idade a $4.340 \mathrm{kcal} \mathrm{kg}^{-1}$ para Eucalyptus grandis com 3 anos de idade.

Os resultados de $\rho_{\mathrm{b} \text { pond }}$ apresentados na Tabela 3 estão dentro do intervalo que Quirino et al. (2005) relataram para 108 espécies florestais, que variaram de 0,200 a $1,080 \mathrm{~g} \mathrm{~cm}^{-3}$. Esses valores também corroboram os observados por Vital e Della Lucia (1987), que não encontraram efeito significativo do espaçamento na $\rho_{\mathrm{b}}$ da madeira. No entanto, são divergentes dos

Revista Árvore, Viçosa-MG, v.38, n.3, p.551-559, 2014 
encontrados por Roque e Ledzema (2003), que verificaram aumento da $\rho_{\mathrm{b}}$ com o incremento do espaçamento em madeira de Tectona grandis. Em contrapartida, Garcia et al. (1991) relataram diminuição da $\rho_{b}$ da madeira com o aumento do espaçamento em madeiras de Eucalyptus grandis e Eucalyptus saligna. Para Goulart et al. (2003), essas divergências de resultados podem ser decorrentes de diversos fatores, como a variabilidade genética dos povoamentos originados de sementes.

Pode-se observar que houve variação na $\rho_{\mathrm{b} \text { pond }}$ entre árvores de mesma espécie, sendo as maiores amplitudes encontradas nas espécies Acacia mearnsii e Ateleia glazioviana. Para Trugilho et al. (1996), as causas dessa característica na $\rho_{b}$ podem ser atribuídas às diferentes condições ambientais e genéticas de cada árvore ou, ainda, em função de diferentes idades. Como as espécies estudadas estavam submetidas a condições ambientais e idades iguais, o fator genético deve ser a possível causa dessa variação. Para Alzate et al. (2005), esses fatores são os principais causadores da variabilidade da $\rho_{\mathrm{b}}$ em espécies arbóreas.

Alguns trabalhos na literatura relatam a $\rho_{\mathrm{b}}$ de diferentes espécies florestais. Jesus e Vital (1986), estudando árvores de Eucalyptus grandis com 7 e 8 anos de idade, evidenciaram que a média dessa característica tecnológica foi igual a $0,460 \mathrm{~g} \mathrm{~cm}^{-3}$. Já Alzate et al. (2005), trabalhando com a mesma espécie aos 3,5 anos de idade, concluíram que a média foi de $0,440 \mathrm{~g} \mathrm{~cm}^{-3}$. Trevisan et al. (2012), estudando a influência do desbaste em árvores centrais de Eucalyptus grandis com idades de 4 a 18 anos, encontraram variação dos valores médios de $\rho_{\mathrm{b}}$ de 0,390 a $0,462 \mathrm{~g} \mathrm{~cm}^{-3}$, confirmando a existência de variabilidade dentro da espécie.

Em estudos realizados por Pauleski (2010), com árvores do extrato médio e dominantes de Pinus taeda, ele observou que, à medida que os espaçamentos entre linhas aumentavam, a $\rho_{\mathrm{b}}$ apresentava a mesma variação. Chies (2005), estudando Pinus taeda, observou que a massa específica básica apresentou valores decrescentes com o aumento do espaçamento entre árvores, e os valores variaram entre 0,434 e 0,414 $\mathrm{g} \mathrm{cm}^{-3}$. Em contrapartida, Castelo (2007) concordou que a massa específica básica decresce com o aumento do espaçamento. Já Montagna et al. (1980) descartaram a influência do espaçamento na massa específica básica, em seus estudos com Pinus caribaea var. hondurensis, com 17 anos de idade, plantados em três diferentes espaçamentos, destacando que a massa específica básica não variou com os espaçamentos adotados.

Da mesma forma, Brito e Barrichello (1980) relataram que madeiras que apresentam $\rho_{\mathrm{b}}$ maior produzem carvão vegetal mais denso, e madeiras mais leves resultam em carvões mais leves e porosos. Segundo Vale et al. (2002), ao se utilizarem madeira com baixa $\rho_{\mathrm{b}}$ para a produção direta de calor, haverá queima rápida e menor produção de energia por unidade de volume, ao contrário do que ocorre com madeiras com maiores $\rho_{b}$, porém estas apresentam maiores dificuldades para início da queima. Esses mesmos autores sugeriram a faixa intermediária entre madeiras médias e duras, variando a $\rho_{\mathrm{b}}$ de 0,650 a $0,800 \mathrm{~g} \mathrm{~cm}^{-3}$ para utilização da madeira na forma de lenha, com o intuito de facilitar o início da queima.

Com relação a um produto energético em específico, o carvão vegetal, a $\rho_{\mathrm{b}}$ da madeira, se correlaciona positivamente com a sua densidade, ou seja, quanto mais densa for a madeira, mais denso será o carvão vegetal (BRITO; BARRICHELO, 1980). Para Andrade e Carvalho (1998), elevado gradiente de densidade entre as regiões da casca e da medula de árvores de maior diâmetro proporciona maior presença de fissuras e trincas no material produzido. Dessa forma, esses autores indicaram o uso de árvores de menor diâmetro para melhor qualidade de carvão vegetal.

\section{CONCLUSÕES}

Os PCS de todas as espécies no primeiro ano após o plantio foram superiores aos do terceiro ano, destacando-se a Acacia mearnsii, com as maiores médias nos períodos estudados.

A $\rho_{\mathrm{b} \text { pond }}$ da madeira não apresentou variação sistemática de aumento ou redução ao longo do tempo, e as espécies Acacia mearnsii e Ateleia glazioviana exibiram os maiores valores dessa propriedade.

Os diferentes espaçamentos de plantio não induziram a variação do PCS e da $\rho_{\mathrm{b} \text { pond }}$ nas espécies estudadas, não sendo verificada variação sistemática em relação ao espaço vital proporcionado pelo espaçamento.

Revista Árvore, Viçosa-MG, v.38, n.3, p.551-559, 2014

\section{Silf}

Revista Arvore, Viçosa-MG, v.38, n.3, p.5, 


\section{REFERÊNCIAS}

ALZATE, S. B. A. et al. Variação longitudinal da densidade básica da madeira de clones de Eucalyptus grandis Hill ex Maiden, E. saligna Sm. e E. grandis x urophylla. Scientia Forestalis, n.68, p.87-95, 2005.

ASSOCIAÇÃO BRASILEIRA DE NORMAS TÉCNICAS - ABNT. Determinação do poder calorífico superior, NBR 8633. Rio de Janeiro: 1984. 13p.

ANDRADE, A. M.; CARVALHO, L. M. Potencialidades energéticas de oito espécies florestais do estado do Rio de Janeiro. Floresta e Ambiente, v. 5, n. 1, p. 24-42, 1998.

BAgGiO, A. J. Timbó: uma alternativa para a produção perene de adubo verde. Colombo: Embrapa Florestas, 2002. 8p. (Circular Técnica, 68)

BRASIL. Empresa de Pesquisa Energética.

Balanço Energético Nacional 2012: ano base 2011: Resultados Preliminares. Rio de Janeiro: 2012. 43p.

BRITO J. O.; BARRICHELO L. E. G. Correlações entre características físicas e químicas da madeira e a produção de carvão: 2 - Densidade da madeira $\mathrm{x}$ densidade do carvão. IPEF, n. 20, p.121-126, 1980.

CARVALHO, P. E. R. Espécies introduzidas alternativas às dos gêneros Pinus e Eucalyptus para reflorestamento no centro-sul do Brasil. In: SEMINÁRIO ESPÉCIES NÃO TRADICIONAIS PARA PLANTIOS COM FINALIDADES PRODUTIVAS E AMBIENTAIS, 1998, Curitiba. Anais... Curitiba: EMBRAPA-CNPF, 1998. p.75-99.

CASTELO, P. A. R. Avaliação da qualidade da madeira de Pinus taeda em diferentes sítios de crescimento e espaçamentos, através do método não destrutivo de emissão de ondas de tensão. 2007. $151 \mathrm{f}$. Tese (Doutorado em Ciências Florestais) -

Universidade Federal do Paraná, Curitiba, 2007.

ÇENGEL, Y. A.; BOLES, M. A.

Termodinâmica. 5.ed. São Paulo: McGrawHill, 2006. 740p.

Revista Árvore, Viçosa-MG, v.38, n.3, p.551-559, 2014
CHIES, D. Influência do espaçamento sobre a qualidade e o rendimento da Madeira serrada de Pinus taeda L. 2005. 123f. Dissertação (Mestrado em Ciências Florestais) - Universidade Federal do Paraná, Curitiba, 2005.

CINTRA, T. C. Avaliações energéticas de espécies florestais nativas plantadas na região do Médio Paranapanema, SP. 2009. 85f. Dissertação (Mestrado em Recursos Florestais) - Escola Superior de Agricultura "Luiz de Queiroz”, Piracicaba, 2009.

\section{COMISSION PANAMERICANA DE NORMAS}

TECNICAS. Maderas: método de determinación del peso especifico aparente: COPANT 30:1-004. Caracas: $1971.7 \mathrm{p}$.

CUNHA, M. P. S. C. et al. Estudo químico de 55 espécies lenhosas para geração de energia em caldeiras. In: ENCONTRO BRASILEIRO EM MADEIRAS E EM ESTRUTURAS DE MADEIRA, 3., 1989, São Carlos. Anais... São Carlos: 1989. v.2. p.93-121.

FINGER, C. A. G. Fundamentos de biometria florestal. Santa Maria: UFSM/ CEPEF/FATEC, 2002. p.269.

GARCIA, C. H. et al. Comportamento florestal do Eucalyptus grandis e Eucalyptus saligna em diferentes espaçamentos. IPEF, n.179, p.1-8, 1991. (Circular Técnica).

GOULART, M. et al. Massa específica básica e massa seca de madeira de Eucalyptus grandis sob o efeito do espaçamento de plantio e da posição axial no tronco. Ciência Florestal, v.13, n.2, p.167-175, 2003.

HOWARD, A. M. Heat of combustion of various southern pine materials. Wood Science, v.5, n.3, p.194-197, 1973.

JANKOWSKY, I. P. et al. Madeiras brasileiras. Caxias do Sul: Spectrum, 1990. 172p.

JARA, E. R. P. O poder calorífico de algumas madeiras que ocorrem no Brasil. São Paulo: Instituto de Pesquisas Tecnológicas - IPT, 1989. (Comunicação Técnica, 1797). 
JESUS, R. M.; VITAL, B. R. Comparação entre densidades de matrizes e de clones de Eucalyptus grandis. Revista Árvore, v.10, n.1, p.82-90, 1986.

LOPES, M. C. Agrupamento de árvores matrizes de Eucalyptus grandis em função das variáveis dendrométricas e das características tecnológicas da madeira. 2003. 93f. Dissertação (Mestrado em Engenharia Florestal) - Universidade Federal de Santa Maria, Santa Maria, 2003.

MALUF, J. R. T. Nova classificação climática do Estado do Rio Grande do Sul. Revista

Brasileira de Agrometeorologia, v.8, n.1, p.141-150, 2000.

MATTOS P. P. Caracterização física, química e anatômica da madeira de timbó (Ateleia glazioveana). Colombo: Embrapa Florestas, 2000. 6p. (Comunicado Técnico, 47).

MONTAGNA, R. G. et al. Peso de matéria seca, densidade básica e dimensões de fibras de Pinus caribea Mor. var. hondurensis Bar. Et Golf.. Silvicultura em São Paulo, v.13/ 14, p.23-32, 1980.

MÜlleR, M. D. Produção de madeira para geração de energia elétrica numa plantação clonal de eucalipto em Etamarandiba, MG. 2005. 94f. Tese (Doutorado em Engenharia Florestal) Universidade Federal de Viçosa. Viçosa, MG, 2005.

PAULESKI, D. T. Influência do espaçamento sobre o crescimento e a qualidade da madeira de Pinus taeda L.. 2010. 198f. Tese (Doutorado em Engenharia Florestal) - Universidade Federal de Santa Maria, Santa Maria, 2010.

PEREIRA, J. C. D. et al. Características da madeira de algumas espécies de eucalipto plantadas no Brasil. Colombo: Embrapa Florestas, 2000. v.1. 113p.
QUIRINO, W. F. et al. Poder calorífico da madeira e de materiais ligno-celulósicos. Revista da Madeira. v.15, n.89, p.100-106, 2005.

ROQUE, R. M.; LEDZEMA, R. M. Efecto del espaciamiento en plantación sobre dos propiedades físicas de madera de teca a lo largo del fuste. Madera y Bosques, v.9, n.2, p.1527, 2003.

SAS LEARNING EDITION. Getting started with the SAS Learning Edition. Cary: 2003. 200p.

SILVA, D. A. et al. Ponto de amostragem ao longo do fuste para estimativa do poder calorífico da madeira. Ciência Rural, v.42, n.9, p.1588-1595, 2012.

TREVISAN, R. et al. Variação axial e efeito do desbaste na massa específica das árvores centrais de Eucalyptus grandis. Ciência Rural, v.42, n.2, p.312-318, 2012.

TRUGILHO, P. F. et al. Influência da idade nas características físico-químicas e anatômicas da madeira de Eucalyptus saligna. Cerne, v.2, n.1, p.94-111, 1996.

VALE, A. T. et al. Produção de energia do fuste de Eucalyptus grandis Hill ex. Maiden e Acacia mangium Willd em diferentes níveis de adubação. Cerne, v.6, n.1, p.83-88, 2000.

VALE, A. T. et al. Qualificação e caracterização energética da madeira e casca de espécies do cerrado. Ciência Florestal, v.12, n.1, p.71-80, 2002.

VITAL, B. R.; DELLA LUCIA, R. M. Efeito do espaçamento na produção em peso e na qualidade da madeira de Eucalyptus grandis e Eucalyptus saligna aos 52 meses de idade. Revista Árvore, v.11, n.2, p.132-145, 1987.

WASHUSEN, R. et al. Effect of thinning and fertilizer on the cellulose crystallite width of Eucalyptus globulus. Wood Science and Technology, v.39, n.7, p.569-578, 2005. 


\section{INFLUÊNCIA DO ESPAÇAMENTO NAS CARACTERÍSTICAS ENERGÉTICAS DE ESPÉCIES ARBÓREAS EM PLANTIOS DE CURTA ROTAÇÃO}

Página 555, Tabela 1, onde se lê:

Tabela 1 - Análise de variância do poder calorífico superior (PCS) e da massa específica básica ( $\rho_{\mathrm{b}}$ ) da madeira das espécies arbóreas distribuídas nos diferentes espaçamentos, em diferentes anos após o plantio, no Município de Frederico Westphalen, RS.

Table 1 - Analysis of variance for the higher heating value (PCS) and basic density $\left(\rho_{b}\right)$ of wood in the tree species distributed in different spacings, in different years after planting, in the city of Frederico Westphalen, $R S$.

\begin{tabular}{|c|c|c|c|c|}
\hline \multirow[t]{2}{*}{ Espécie } & \multicolumn{4}{|c|}{ Espaçamento (m) } \\
\hline & $2,0 \times 1,0$ & $2,0 \times 1,5$ & $3,0 \times 1,0$ & $3,0 \times 1,5$ \\
\hline & \multicolumn{4}{|c|}{ Ano 1} \\
\hline A. mearnsii & 0,415 a & 0,402 a & 0,406 b & $0,405 \mathrm{a}$ \\
\hline M. scabrella & $0,388 \mathrm{~b}$ & $0,409 a b$ & $0,405 \mathrm{~b}$ & 0,406 a \\
\hline E. grandis & $0,382 \mathrm{~b}$ & $0,387 \mathrm{~b}$ & $0,390 \mathrm{~b}$ & 0,391 a \\
\hline A. glazioviana & 0,417 a & 0,423 a & 0,440 a & 0,399 a \\
\hline \multirow[t]{2}{*}{ CV (\%) } & 4,52 & 3,70 & 5,15 & 1,72 \\
\hline & \multicolumn{4}{|c|}{ Ano 3} \\
\hline A. mearnsii & $0,447 \mathrm{ab}$ & $0,414 \mathrm{~b}$ & 0,414 a & $0,436 \mathrm{ab}$ \\
\hline M. scabrella & $0,405 \mathrm{~b}$ & $0,396 \mathrm{~b}$ & 0,407 b & $0,411 \mathrm{~b}$ \\
\hline E. grandis & 0,367 с & $0,371 \mathrm{c}$ & 0,365 с & $0,384 \mathrm{~b}$ \\
\hline A. glazioviana & 0,472 a & 0,468 a & 0,489 а & 0,486 a \\
\hline CV (\%) & 10,96 & 9,98 & 12,32 & 10,11 \\
\hline
\end{tabular}

Em que * = significativo a 5\% de probabilidade de erro, conforme a distribuição de Fisher; ${ }^{\text {ns }}=$ não significativo a $5 \%$ de probabilidade de erro, conforme a distribuição de Fisher.

deve-se ler :

Tabela 1 - Análise de variância do poder calorífico superior (PCS) e da massa específica básica ( $\rho_{\mathrm{b}}$ ) da madeira das espécies arbóreas distribuídas nos diferentes espaçamentos, em diferentes anos após o plantio, no Município de Frederico Westphalen, RS.

Table 1 - Analysis of variance for the higher heating value (PCS) and basic density $\left(\rho_{b}\right)$ of wood in the tree species distributed in different spacings, in different years after planting, in the city of Frederico Westphalen, RS.

\begin{tabular}{|c|c|c|c|c|}
\hline \multicolumn{5}{|c|}{ Efeito principal } \\
\hline \multirow{2}{*}{\multicolumn{2}{|c|}{ Fator de estudo }} & \multirow[t]{2}{*}{ GL } & \multicolumn{2}{|c|}{ Quadrado médio } \\
\hline & & & PCS & $\rho_{\mathrm{b}}$ \\
\hline Espécie & & 3 & $223042 *$ & $0,0598 *$ \\
\hline Espaçam & & 3 & $7908^{\mathrm{ns}}$ & 0,0018 \\
\hline Espécie & & 9 & 4672 ns & $0,0009 \mathrm{~ns}$ \\
\hline \multicolumn{5}{|c|}{ * Significativo à probabilidade de erro tipo A } \\
\hline Ano & & 1 & 1205568* & $0,0211 *$ \\
\hline Ano $x$ es & & 3 & 51823* & $0,0182 *$ \\
\hline Ano $x$ es & & 3 & 7272 ns & $0,0011 \mathrm{~ns}$ \\
\hline Ano $x$ es & & 9 & 4989 ns & $0,0007^{\mathrm{ns}}$ \\
\hline \multicolumn{5}{|c|}{ * Significativo à probabilidade de erro tipo B } \\
\hline Coeficie & $(\%)$ & & $86 \%$ & $61 \%$ \\
\hline Coeficie & & & 1,67 & 7,03 \\
\hline \multicolumn{5}{|c|}{ Efeito simples } \\
\hline \multicolumn{5}{|c|}{ Ano $x$ espécie } \\
\hline \multirow[t]{2}{*}{ Ano } & 1 & 3 & $146548 *$ & $0,0063 *$ \\
\hline & 3 & 3 & $128318^{*}$ & $0,0701 *$ \\
\hline \multirow[t]{4}{*}{ Espécie } & A. mearnsii & 1 & $375500^{*}$ & $0,0083^{*}$ \\
\hline & M. scabrella & 1 & $416172 *$ & $0,0002 \mathrm{~ns}$ \\
\hline & E. grandis & 1 & $523272 *$ & $0,0045^{*}$ \\
\hline & A. glazioviana & 1 & $46095 *$ & $0,0599 *$ \\
\hline
\end{tabular}

Em que * = significativo a 5\% de probabilidade de erro, conforme a distribuição de Fisher; ${ }^{\text {ns }}=$ não significativo a $5 \%$ de probabilidade de erro, conforme a distribuição de Fisher.

Revista Árvore, Viçosa-MG, v.38, n.3, p.551-559, 2014 
Página 556, Tabela 2, onde se lê:

Tabela 2 - Poder calorífico superior (PCS), em kcal kg-1 , das espécies arbóreas distribuídas nos diferentes espaçamentos, em diferentes anos após o plantio, no Município de Frederico Westphalen, RS.

Table 2 - Higher heating value (PCS), in $\mathrm{kcal} \mathrm{kg}^{-1}$, of the tree species distributed in different spacings, in different years after planting, in the city of Frederico Westphalen-RS.

\begin{tabular}{|c|c|c|c|c|}
\hline \multicolumn{5}{|c|}{ Efeito principal } \\
\hline \multirow{2}{*}{\multicolumn{2}{|c|}{ Fator de estudo }} & \multirow[t]{2}{*}{ GL } & \multicolumn{2}{|c|}{ Quadrado médio } \\
\hline & & & PCS & $\rho_{\mathrm{b}}$ \\
\hline Espécie & & 3 & $223042 *$ & $0,0598 *$ \\
\hline Espaçam & & 3 & 7908 ns & $0,0018 \mathrm{~ns}$ \\
\hline Espécie & & 9 & 4672 ns & 0,0009 ns \\
\hline \multicolumn{5}{|c|}{ * Significativo à probabilidade de erro tipo A } \\
\hline$\overline{\text { Ano }}$ & & 1 & 1205568* & $0,0211^{*}$ \\
\hline Ano $x$ es & & 3 & $51823 *$ & $0,0182 *$ \\
\hline Ano $x$ es & & 3 & $7272 \mathrm{~ns}$ & $0,0011 \mathrm{~ns}$ \\
\hline Ano $x$ es & & 9 & 4989 ns & $0,0007^{\text {ns }}$ \\
\hline \multicolumn{5}{|c|}{ * Significativo à probabilidade de erro tipo B } \\
\hline Coeficie & $(\%)$ & & $86 \%$ & $61 \%$ \\
\hline Coeficie & & & 1,67 & 7,03 \\
\hline \multicolumn{5}{|c|}{ Efeito simples } \\
\hline \multicolumn{5}{|c|}{ Ano $x$ espécie } \\
\hline \multirow[t]{2}{*}{ Ano } & 1 & 3 & $146548 *$ & $0,0063^{*}$ \\
\hline & 3 & 3 & $128318^{*}$ & $0,0701 *$ \\
\hline \multirow[t]{4}{*}{ Espécie } & A. mearnsii & 1 & $375500^{*}$ & $0,0083^{*}$ \\
\hline & M. scabrella & 1 & $416172 *$ & 0,0002 ns \\
\hline & E. grandis & 1 & $523272 *$ & $0,0045 *$ \\
\hline & A. glazioviana & 1 & $46095^{*}$ & $0,0599 *$ \\
\hline
\end{tabular}

Médias seguidas por letras iguais, na coluna, não diferem entre si, a 5\% de probabilidade de erro, conforme a distribuição de Tukey.

deve-se ler :

Tabela 2 - Poder calorífico superior (PCS), em kcal kg-1 , das espécies arbóreas distribuídas nos diferentes espaçamentos, em diferentes anos após o plantio, no Município de Frederico Westphalen, RS.

Table 2 - Higher heating value (PCS), in kcal kg-1, of the tree species distributed in different spacings, in different years after planting, in the city of Frederico Westphalen-RS.

\begin{tabular}{|c|c|c|c|c|}
\hline \multirow[t]{2}{*}{ Espécie } & \multicolumn{4}{|c|}{ Espaçamento (m) } \\
\hline & $2,0 \times 1,0$ & $2,0 \times 1,5$ & $3,0 \times 1,0$ & $3,0 \times 1,5$ \\
\hline \multicolumn{5}{|c|}{ Ano 1} \\
\hline A. mearnsii & 4726 a & 4788 а & 4792 а & 4828 а \\
\hline M. scabrella & $4647 \mathrm{ab}$ & $4658 \mathrm{~b}$ & $4631 \mathrm{~b}$ & $4679 \mathrm{~b}$ \\
\hline E. grandis & 4531 c & 4600 bc & 4539 bc & $4653 \mathrm{~b}$ \\
\hline A. glazioviana & 4586 bc & 4533 c & $4476 \mathrm{c}$ & $4520 \mathrm{c}$ \\
\hline CV (\%) & 1,81 & 2,33 & 2,98 & 2,70 \\
\hline \multicolumn{5}{|c|}{ Ano 3} \\
\hline A. mearnsii & 4558 a & 4442 a & 4560 a & 4577 a \\
\hline M. scabrella & 4372 b & 4349 ab & $4406 \mathrm{~b}$ & 4435 b \\
\hline E. grandis & $4338 \mathrm{~b}$ & $4289 \mathrm{~b}$ & $4241 \mathrm{c}$ & $4273 \mathrm{c}$ \\
\hline A. glazioviana & 4448 ab & 4441 а & 4440 ab & 4436 b \\
\hline CV (\%) & 2,20 & 1,71 & 2,98 & 2,80 \\
\hline
\end{tabular}

Médias seguidas por letras iguais, na coluna, não diferem entre si, a 5\% de probabilidade de erro, conforme a distribuição de Tukey.

Revista Árvore, Viçosa-MG, v.38, n.3, p.551-559, 2014 
Página 556, Tabela 3, onde se lê:

Tabela 3 -Massa específica básica ponderada $\left(\rho_{\mathrm{b} \text { pond }}\right)$ da madeira, em $\mathrm{g} \mathrm{cm}^{-3}$, das espécies arbóreas distribuídas nos diferentes espaçamentos, em diferentes anos após o plantio, no Município de Frederico Westphalen, RS.

Table 3 - Basic Density weighted ( $\rho_{\text {bpond }}$ ) of wood, in $\mathrm{g} \mathrm{cm}^{-3}$, of the tree species distributed in different spacings, in different years after planting, in the city of Frederico Westphalen, $R S$.

\begin{tabular}{lcccc}
\hline Espécie & \multicolumn{4}{c}{ Espaçamento (m) } \\
\cline { 2 - 5 } & $2,0 \times 1,0$ & $2,0 \times 1,5$ & $3,0 \times 1,0$ & $3,0 \times 1,5$ \\
\hline A. mearnsii & $4726 \mathrm{a}$ & $4788 \mathrm{a}$ & $4792 \mathrm{a}$ & $4828 \mathrm{a}$ \\
M. scabrella & $4647 \mathrm{ab}$ & $4658 \mathrm{~b}$ & $4631 \mathrm{~b}$ & $4679 \mathrm{~b}$ \\
E. grandis & $4531 \mathrm{c}$ & $4600 \mathrm{bc}$ & $4653 \mathrm{~b}$ \\
A. glazioviana & $4586 \mathrm{bc}$ & $4533 \mathrm{c}$ & $4520 \mathrm{c}$ \\
CV (\%) & 1,81 & 2,33 & 2,98 & 2,70 \\
\hline & & & $4576 \mathrm{c}$ \\
\hline A. mearnsii & $4558 \mathrm{a}$ & $4560 \mathrm{a}$ & $4435 \mathrm{~b}$ \\
M. scabrella & $4372 \mathrm{~b}$ & $4442 \mathrm{a}$ & $4273 \mathrm{c}$ \\
E. grandis & $4338 \mathrm{~b}$ & $4349 \mathrm{ab}$ & $4406 \mathrm{~b}$ & $4436 \mathrm{~b}$ \\
A. glazioviana & $4448 \mathrm{ab}$ & $4289 \mathrm{~b}$ & $4241 \mathrm{c}$ & 2,80 \\
CV (\%) & 2,20 & $4441 \mathrm{a}$ & $4440 \mathrm{ab}$ & 2,98 \\
\hline
\end{tabular}

Médias seguidas por letras iguais, na coluna, não diferem entre si, a 5\% de probabilidade de erro, conforme a distribuição de Tukey.

deve-se ler :

Tabela 3 -Massa específica básica ponderada $\left(\rho_{\mathrm{b} \text { pond }}\right)$ da madeira, em $\mathrm{g} \mathrm{cm}^{-3}$, das espécies arbóreas distribuídas nos diferentes espaçamentos, em diferentes anos após o plantio, no Município de Frederico Westphalen, RS.

Table 3 - Basic Density weighted ( $\rho_{\text {ppond }}$ ) of wood, in $\mathrm{g} \mathrm{cm}^{-3}$, of the tree species distributed in different spacings, in different years after planting, in the city of Frederico Westphalen, $R S$.

\begin{tabular}{lcccc}
\hline Espécie & \multicolumn{4}{c}{ Espaçamento $(\mathrm{m})$} \\
\cline { 2 - 5 } & $2,0 \times 1,0$ & $2,0 \times 1,5$ & $3,0 \times 1,0$ & $3,0 \times 1,5$ \\
\hline A. mearnsii & $0,415 \mathrm{a}$ & Ano 1 & $0,405 \mathrm{a}$ \\
M. scabrella & $0,388 \mathrm{~b}$ & $0,402 \mathrm{a}$ & $0,406 \mathrm{~b}$ & $0,406 \mathrm{a}$ \\
E. grandis & $0,382 \mathrm{~b}$ & $0,409 \mathrm{ab}$ & $0,395 \mathrm{~b} \mathrm{~b}$ & $0,391 \mathrm{a}$ \\
A. glazioviana & $0,417 \mathrm{a}$ & $0,387 \mathrm{~b}$ & $0,440 \mathrm{a}$ & $0,399 \mathrm{a}$ \\
CV (\%) & 4,52 & $0,423 \mathrm{a}$ & 5,15 & 1,72 \\
\hline \multicolumn{7}{c}{ mearnsii } & 3,70 & Ano 3 & $0,436 \mathrm{ab}$ \\
M. scabrella & $0,447 \mathrm{ab}$ & $0,414 \mathrm{~b}$ & $0,414 \mathrm{a}$ & $0,411 \mathrm{~b}$ \\
E. grandis & $0,405 \mathrm{~b}$ & $0,396 \mathrm{~b}$ & $0,407 \mathrm{~b}$ & $0,384 \mathrm{~b}$ \\
A. glazioviana & $0,367 \mathrm{c}$ & $0,371 \mathrm{c}$ & $0,365 \mathrm{c}$ & $0,486 \mathrm{a}$ \\
CV (\%) & $0,472 \mathrm{a}$ & $0,468 \mathrm{a}$ & 12,32 & 10,11 \\
\hline
\end{tabular}

Médias seguidas por letras iguais, na coluna, não diferem entre si, a 5\% de probabilidade de erro, conforme a distribuição de Tukey.

Revista Árvore, Viçosa-MG, v.38, n.3, p.551-559, 2014 